\title{
Asymptotic behaviour of curvature and matter in the Penrose limit
}

\author{
Kerstin E. Kunze ${ }^{1}$ \\ Física Teórica, Universidad de Salamanca, \\ Plaza de la Merced s/n, 37008 Salamanca, Spain.
}

\begin{abstract}
The asymptotic behaviour of the components of the Weyl tensor and of the energy-momentum tensor in the Penrose limit is determined. In both cases a peeling-off property is found. Examples of different types of matter are provided. The expansion and shear of the congruence of null geodesics along which the Penrose limit is taken are determined. Finally, the approach to the singularity in the Penrose limit of cosmological space-times is discussed.
\end{abstract}

\section{Introduction}

Recent developments in string-/M theory have let to renewed interest in an argument by Penrose [1] that in a neighbourhood of a null geodesic, which contains no conjugate points, any space-time has a plane wave as a limit (2] and references therein). The Penrose limit construction involves changing first to a suitable system of coordinates and then rescaling by a constant parameter $\Omega$ the coordinates as well as the metric. In the limit $\Omega \rightarrow 0$ a plane wave space-time is obtained. The parameter $\Omega$ can be interpreted as an additional coordinate. Thus lifting an $n$-dimensional space-time to an $(n+1)$ - dimensional space-time with boundary, whose boundary at $\Omega=0$ is given by the Penrose limit [1].

The Penrose limit construction allows to associate any space-time with a plane wave space-time. Another situation in which a space-time approaches a plane wave is in the case of a metric far away from an isolated radiating source [3]. In this case the space-time approaches globally a plane wave whereas in the case of the Penrose limit the plane wave is local in the neighbourhood of a particular geodesic.

In the case of the limiting space-time far away from an isolated radiating source the approach to the limiting plane wave space-time has been determined. Namely, the components of the Weyl tensor show a typical peeling-off behaviour [3. In the Newman-Penrose formalism the components of the Weyl tensor are described by five complex scalars $\Psi_{i}$. In this particular case, as the far field limit is approached these five components become negligible one after the other until only $\Psi_{4}$ remains which is associated with a plane wave space-time. A peeling-off behaviour was also found in space-times admitting two space-like Killing vectors [4. Furthermore, space-times describing light like signals propagating through a general Bondi-Sachs space-time have peeling properties as

\footnotetext{
${ }^{1}$ E-mail: Kerstin.Kunze@cern.ch
} 
well. In certain cases these are different from those of a Bondi-Sachs space-time [5].

Here the asymptotic approach in the Penrose limit to a plane wave will be investigated. Locally any space-time approaches a plane wave along a null geodesic. Thus this is a general feature of any metric. Similarly to the global plane wave limit of the isolated radiating source space-times and those admitting two space-like Killing vectors, a peeling-off behaviour of the curvature as well as the components of the energy-momentum tensor is found in the Penrose limit.

Plane wave space-times are exact classical string vacua [6]. Space-times in general are no such solutions, even though vacuum solutions of general relativity are always solutions to string theory in the low energy limit. Thus the approach to the Penrose limit might give corrections to exact classical string vacua.

In section 2, the Penrose limit construction is formulated using the Newman-Penrose formalism which is especially adapted to the treatment of null geodesics. Then the peeling-off property of the Weyl tensor and of the energy-momentum tensor is found. In section 3 the kinematics of the null geodesics is determined and the approach to null power law singularities is discussed. Finally, in section 4 conclusions are presented.

\section{The Weyl tensor and energy-momentum tensor in the Penrose limit}

Following [1] the metric in the neighbourhood of a segment of a null geodesic containing no conjugate points can be written as

$$
d s^{2}=d u d v+\alpha d v^{2}+\beta_{1} d x d v+\beta_{2} d y d v-\frac{2 e^{-\mu_{1}}}{Z+\bar{Z}}(d x+i Z d y)(d x-i \bar{Z} d y),
$$

where $\alpha, \beta_{i}, Z$ and $\mu_{1}$ are functions of all coordinates $u, v, x, y . Z$ is a complex function. Complex conjugation is denoted by a bar. The parametrization of the components of the metric over the two-dimensional $x-y$ subspace is of the form used in colliding plane wave space-times [7, 8]. Whereas in the case of collding plane waves $Z$ is just a function of $u$ and $v$, here $Z$ is a function of all variables. This form of the metric seems to be convenient, since plane waves, and thus the resulting metrics in the Penrose limit, are a particular solution of colliding plane wave space-times.

Recently, the Penrose limit has been formulated in a covariant form by making use of a paralleltransported frame along the chosen null geodesic [9, 10, 11. Such a frame is obtained by introducing a null tetrad with two real null vectors $l^{\mu}, n^{\mu}$ and two complex null vectors which are complex conjugates of each others $m^{\mu}, \bar{m}^{\mu}$. They satisfy the relations [12, 7]

$$
l_{\mu} n^{\mu}=1, \quad m_{\mu} \bar{m}^{\mu}=-1, \quad g_{\mu \nu}=l_{\mu} n_{\nu}+n_{\mu} l_{\nu}-m_{\mu} \bar{m}_{\nu}-\bar{m}_{\mu} m_{\nu} .
$$

Explicit expressions for the null tetrad and the spin coefficients in the Newman-Penrose formalism are given in the appendix.

The Penrose limit uses the freedom of rescaling coordinates by a constant parameter. Namely, new coordinates $U, V, X, Y$ are introduced as follows [1]

$$
u=U, \quad v=\Omega^{2} V, \quad x=\Omega X, \quad y=\Omega Y,
$$

where $\Omega>0$ is a constant parameter. Furthermore, a conformal transformation is applied to the metric (2.1) such that

$$
d s_{\Omega}^{2}=\Omega^{-2} d s^{2}
$$


resulting in

$$
d s_{\Omega}^{2}=d U d V+\Omega^{2} A d V^{2}+\Omega B_{1} d X d V+\Omega B_{2} d Y d V-\frac{2 e^{-M}}{\zeta+\bar{\zeta}}(d X+i \zeta d Y)(d X-i \bar{\zeta} d Y),
$$

where $A, B_{i}$ and $\zeta$ are functions of $U, V, X, Y$ and replace $\alpha, \beta_{i}$ and $Z$, respectively, of the metric (2.1).

The Penrose limit is defined as

$$
\hat{d s}^{2}=\lim _{\Omega \rightarrow 0} d s_{\Omega}^{2}
$$

Applying this to the metric (2.5) a plane wave metric is obtained. The approach to this plane wave, in powers of $\Omega$, will be studied by means of the components of the Weyl tensor. Furthermore, for non-vacuum space-times the asymptotic behaviour of the components of the energy-momentum tensor will be discussed.

In the Newman-Penrose formalism the ten components of the Weyl tensor are encoded in five complex scalars $\Psi_{i}$ (cf appendix). These involve the spin coefficients as well as their directional derivatives. The Ricci identities [12] together with the asymptotic behaviour of the spin coefficients determine the scaling with the Penrose parameter $\Omega$ of the components of the Weyl tensor.

Using the expressions for the spin coefficients as given in the appendix (cf equation (5.40) for the rescaled metric (2.5) leads to the following overall scaling with the Penrose parameter $\Omega$, which determines the factor $\Omega^{n}$ in front of the $U$ - dependent part,

$$
\begin{array}{llrl}
\kappa(\Omega)=\mathcal{O}\left(\Omega^{3}\right) & \sigma(\Omega)=\mathcal{O}\left(\Omega^{2}\right) & \lambda(\Omega)=\mathcal{O}\left(\Omega^{0}\right) & \nu=0 \\
\rho(\Omega)=\mathcal{O}\left(\Omega^{2}\right) & \mu(\Omega)=\mathcal{O}\left(\Omega^{0}\right) & \tau(\Omega)=\mathcal{O}(\Omega) & \pi(\Omega)=\mathcal{O}(\Omega) \\
\epsilon(\Omega)=\mathcal{O}\left(\Omega^{2}\right) & \gamma(\Omega)=\mathcal{O}\left(\Omega^{0}\right) & \alpha_{N P}(\Omega)=\mathcal{O}(\Omega) & \beta(\Omega)=\mathcal{O}(\Omega) .
\end{array}
$$

The scaling with $\Omega$ of the directional derivatives (cf appendix equation (5.41) ) is found by going back to the original coordinates $u, v, x, y$ since all metric functions are functions of these coordinates and taking partial derivatives with respect to the new coordinates $U, V, X, Y$ leads to derivatives with respect to the old coordinates with an additional factor of $\Omega$. For example,

$$
D(\Omega)=-\Omega^{2} \frac{\alpha}{2} \frac{\partial}{\partial u}+\Omega^{2} \frac{\partial}{\partial v},
$$

where $\alpha$ is, as before, a function of $u, v, x, y$. Therefore, the directional derivatives scale as follows

$$
D(\Omega)=\mathcal{O}\left(\Omega^{2}\right) \quad \Delta(\Omega)=\mathcal{O}\left(\Omega^{0}\right) \quad \delta(\Omega)=\mathcal{O}(\Omega) \quad \bar{\delta}(\Omega)=\mathcal{O}(\Omega) .
$$

Using the following Ricci identities [12] and the expression for $\Psi_{2}$ derived from Ricci identities

$$
\begin{aligned}
D \sigma-\delta \kappa= & \sigma(3 \epsilon-\bar{\epsilon}+\rho+\bar{\rho})+\kappa\left(\bar{\pi}-\tau-3 \beta-\bar{\alpha}_{N P}\right)+\Psi_{0} \\
D \beta-\delta \epsilon= & \sigma\left(\alpha_{N P}+\pi\right)+\beta(\bar{\rho}-\bar{\epsilon})-\kappa(\mu+\gamma)-\epsilon\left(\alpha_{N P}-\bar{\pi}\right)+\Psi_{1} \\
\Psi_{2}= & \frac{1}{3}\left[D \gamma-\Delta \epsilon-\delta \alpha_{N P}+\bar{\delta} \beta+D \mu-\delta \pi-\left(\alpha_{N P}+\pi\right)(\tau+\bar{\pi}-\bar{\alpha}+\beta)\right. \\
& -\beta(\bar{\tau}+\pi+\alpha-\bar{\beta})+(\mu+\gamma)(\epsilon+\bar{\epsilon}+\rho-\bar{\rho}) \\
& +\epsilon(\gamma+\bar{\gamma}+\mu-\bar{\mu})+2 \nu \kappa-2 \sigma \lambda] \\
\Delta \alpha_{N P}-\bar{\delta} \gamma= & \nu(\rho+\epsilon)-\lambda(\tau+\beta)+\alpha_{N P}(\bar{\gamma}-\bar{\mu})+\gamma(\bar{\beta}-\bar{\tau})-\Psi_{3} \\
\Delta \lambda-\bar{\delta} \nu= & -\lambda(\mu+\bar{\mu}+3 \gamma-\bar{\gamma})+\nu\left(3 \alpha_{N P}+\bar{\beta}+\pi-\bar{\tau}\right)-\Psi_{4}
\end{aligned}
$$


shows that the components of the Weyl tensor scale as

$$
\Psi_{i}(\Omega)=\mathcal{O}\left(\Omega^{4-i}\right),
$$

where $i=0, . ., 4$. Thus the components of the curvature show a peeling-off behaviour. The approach to the plane wave is determined by the constant parameter $\Omega$. This is different from the global peeling-off behaviour found, for example, in space-times describing the far-field of an isolated radiating source. In this case the peeling-off behaviour is controlled by one of the coordinates. Namely, the components of the Weyl tensor behave as [3]

$$
\Psi_{k}=\mathcal{O}\left(r^{k-5}\right),
$$

in the limit $r \rightarrow \infty$ and $r$ is a radial coordinate. Thus the dependence on this coordinate remains whereas this is not the case of the Penrose limit. Here, $\Psi_{4}$ is independent of the parameter $\Omega$. As was already observed in the covariant approach to the Penrose limit as formulated in [9, 10, here the component of the Weyl tensor $\Psi_{4}$ is exactly the same as in the original space-time (2.1).

Using Einstein's equations in the tetrad basis

$$
R_{(a)(b)}-\frac{1}{2} \eta_{(a)(b)} R=-T_{(a)(b)}
$$

allows to determine the asymptotic behaviour of the components of the energy-momentum tensor. In the Newman-Penrose formalism the components of the Ricci tensor are denoted by $\Phi_{a b}$ (cf. appendix equation (5.43) ). The Ricci identities as given in [12], e.g., can be used to find the scaling of $\Phi_{a b}$ and thus the behaviour of the components of the energy-momentum tensor.

The useful Ricci identities to determine the scaling of the $\Phi_{a b}$ are given by 12 ]

$$
\begin{aligned}
D \rho-\bar{\delta} \kappa & =\left(\rho^{2}+\sigma \bar{\sigma}\right)+\rho(\epsilon+\bar{\epsilon})-\bar{\kappa} \tau-\kappa\left(3 \alpha_{N P}+\bar{\beta}-\pi\right)+\Phi_{00} \\
D \alpha_{N P}-\bar{\delta} \epsilon= & \alpha_{N P}(\rho+\bar{\epsilon}-2 \epsilon)+\beta \bar{\sigma}-\bar{\beta} \epsilon-\kappa \lambda-\bar{\kappa} \gamma+\pi(\epsilon+\rho)+\Phi_{10} \\
D \lambda-\bar{\delta} \pi= & \rho \lambda+\bar{\sigma} \mu+\pi\left(\pi+\alpha_{N P}-\bar{\beta}\right)-\nu \bar{\kappa}-\lambda(3 \epsilon-\bar{\epsilon})+\Phi_{20} \\
\delta \nu-\Delta \mu= & \mu^{2}+\lambda \bar{\lambda}+\mu(\gamma+\bar{\gamma})-\bar{\nu} \pi+\nu\left(\tau-3 \beta-\bar{\alpha}_{N P}\right)+\Phi_{22} \\
\delta \gamma-\Delta \beta= & \gamma\left(\tau-\bar{\alpha}_{N P}-\beta\right)+\mu \tau-\sigma \nu-\epsilon \bar{\nu}-\beta(\gamma-\bar{\gamma}-\mu)+\alpha \bar{\lambda}+\Phi_{12} \\
\Delta \rho-\bar{\delta} \tau= & -(\rho \bar{\mu}+\sigma \lambda)+\tau\left(\bar{\beta}-\alpha_{N P}-\bar{\tau}\right)+\rho(\gamma+\bar{\gamma})+\nu \kappa-\Psi_{2}-2 \Lambda \\
\delta \alpha_{N P}-\bar{\delta} \beta= & \mu \rho-\lambda \sigma+\alpha_{N P} \bar{\alpha}_{N P}+\beta \bar{\beta}-2 \alpha \beta+\gamma(\rho-\bar{\rho})+\epsilon(\mu-\bar{\mu})-\Psi_{2} \\
& +\Phi_{11}+\Lambda .
\end{aligned}
$$

For the case of the rescaled metric (2.5) this gives the following scaling behaviour with $\Omega$

$$
\begin{array}{lll}
\Phi_{00}=\mathcal{O}\left(\Omega^{4}\right) & \Phi_{10}=\mathcal{O}\left(\Omega^{3}\right) & \Phi_{20}=\mathcal{O}\left(\Omega^{2}\right) \\
\Phi_{11}=\mathcal{O}\left(\Omega^{2}\right) & \Phi_{12}=\mathcal{O}(\Omega) & \Phi_{22}=\mathcal{O}\left(\Omega^{0}\right) \\
\Lambda=\mathcal{O}\left(\Omega^{2}\right), & &
\end{array}
$$

which determines the remaining components. Thus the component $\Phi_{22}$ is exactly the one of the original space-time (2.1).

Therefore, Einstein's equations imply that the components of the energy-momentum tensor scale as

$$
\begin{array}{llll}
T_{(1)(1)}=\mathcal{O}\left(\Omega^{4}\right) & T_{(1)(3)}=\mathcal{O}\left(\Omega^{3}\right) & T_{(1)(2)}=\mathcal{O}\left(\Omega^{2}\right) & T_{(3)(3)}=\mathcal{O}\left(\Omega^{2}\right) \\
T_{(3)(4)}=\mathcal{O}\left(\Omega^{2}\right) & T_{(2)(3)}=\mathcal{O}(\Omega) & T_{(2)(2)}=\mathcal{O}\left(\Omega^{0}\right) . &
\end{array}
$$


As a first example, consider a space-time containing a Maxwell field, $F_{\mu \nu}$. Its components are given in the Newman-Penrose formalism by [12]

$$
\begin{aligned}
\phi_{0} & =F_{(1)(3)}=F_{\mu \nu} l^{\mu} m^{\nu} \\
\phi_{1} & =\frac{1}{2}\left(F_{(1)(2)}+F_{(4)(3)}\right)=\frac{1}{2} F_{\mu \nu}\left(l^{\mu} n^{\nu}+\bar{m}^{\mu} m^{\nu}\right) \\
\phi_{2} & =F_{(4)(2)}=F_{\mu \nu} \bar{m}^{\mu} n^{\nu} .
\end{aligned}
$$

The non-vanishing components of the energy-momentum tensor are given by [12]

$$
\begin{array}{lll}
T_{(1)(1)}=-2 \phi_{0} \bar{\phi}_{0} & T_{(1)(2)}+T_{(3)(4)}=-4 \phi_{1} \bar{\phi}_{1} & T_{(1)(3)}=-2 \phi_{0} \bar{\phi}_{1} \\
T_{(2)(2)}=-2 \phi_{2} \bar{\phi}_{2} & T_{(2)(3)}=-2 \phi_{1} \bar{\phi}_{2} & T_{(3)(3)}=-2 \phi_{0} \bar{\phi}_{2} .
\end{array}
$$

Thus the components of the Maxwell field scale in the Penrose limit as follows,

$$
\phi_{0}=\mathcal{O}\left(\Omega^{2}\right) \quad \phi_{1}=\mathcal{O}(\Omega) \quad \phi_{2}=\mathcal{O}\left(\Omega^{0}\right),
$$

or equivalently

$$
\phi_{i}=\mathcal{O}\left(\Omega^{2-i}\right),
$$

where $i=0,1,2$. The $\phi_{2}$ component of the Maxwell field is the one of the original space-time (2.1). In comparison, the peeling-off behaviour of the components of the Maxwell tensor in the background of the far field of an isolated, radiating source is given by (see for example, [13]),

$$
\phi_{i}=\mathcal{O}\left(r^{i-3}\right) \text {, }
$$

in the limit $r \rightarrow \infty$ and where $r$ is a radial coordinate.

As a second example, the asymptotic behaviour of the energy-momentum tensor describing a perfect fluid will be discussed. The energy-momentum tensor is given in this case by

$$
T^{\mu \nu}=\left(\rho_{f}+p_{f}\right) u^{\mu} u^{\nu}-p_{f} g^{\mu \nu},
$$

where $\rho_{f}$ and $p_{f}$ are the energy and pressure densities, respectively. Following [7] the velocity can be written in terms of the tetrad vectors as

$$
u^{\mu}=\frac{1}{\sqrt{2}}\left(a l^{\mu}+b n^{\mu}\right)
$$

where $a$ and $b$ are constants. For $a b=1, u^{\mu}$ is a time-like vector field. This describes the matter field in the original space-time, for example, a perfect fluid cosmology. In order to determine the asymptotic behaviour of the energy-momentum tensor, the constants $a$ and $b$ are chosen such that, $a \equiv 1$ and $b \equiv \Omega^{2}$, resulting in $u_{\mu} u^{\mu}=\Omega^{2} \neq 1$. Furthermore, the last term in equation (2.21) is rescaled by a factor $\Omega^{2}$. The non-vanishing components of the energy-momentum tensor are found to be

$$
\begin{array}{ll}
T_{(1)(1)}=\frac{\Omega^{4}}{2}\left(\rho_{f}+p_{f}\right) & T_{(1)(2)}=\frac{\Omega^{2}}{2}\left(\rho_{f}-p_{f}\right) \\
T_{(2)(2)}=\frac{1}{2}\left(\rho_{f}+p_{f}\right) & T_{(3)(4)}=\Omega^{2} p_{f} .
\end{array}
$$


Thus in the limit $\Omega \rightarrow 0$ the fluid becomes null. $T_{(2)(2)}$ is the only non-vanishing component and the scaling follows equation (2.15). Furthermore, as already found in [10] the Penrose limit of a perfect fluid space-time is flat only for $p_{f}+\rho_{f}=0$, that is for a cosmological constant.

Einstein's equations yield the non-vanishing components of the Ricci tensor and the Ricci scalar as

$$
\begin{array}{lll}
\Phi_{00}=\frac{\Omega^{4}}{4}\left(\rho_{f}+p_{f}\right) & \Phi_{11}=\frac{\Omega^{2}}{8}\left(\rho_{f}+p_{f}\right) \\
\Phi_{22}=\frac{1}{4}\left(\rho_{f}+p_{f}\right) & \Lambda=\frac{\Omega^{2}}{24}\left(\rho_{f}-3 p_{f}\right) .
\end{array}
$$

Thus the scaling behaviour follows equation (2.14).

\section{Kinematics of the null congruence}

The focussing behaviour of a congruence of geodesics is described by the Raychaudhuri equation,

$$
\frac{d \theta}{d u}=-\frac{1}{2} \theta^{2}-\sigma_{\alpha \beta} \sigma^{\alpha \beta}+\omega_{\alpha \beta} \omega^{\alpha \beta}-R_{\alpha \beta} k^{\alpha} k^{\beta},
$$

where $k^{\alpha}$ in this case is the tangent vector along a congruence of null geodesics (cf e.g. [14]). This involves the expansion $\theta$, the shear tensor $\sigma_{\alpha \beta}$ and the vorticity tensor $\omega_{\alpha \beta}$ of the geodesics. The Penrose limit of a space-time is calculated along a null geodesic. Choosing this null geodesic to calculate the expansion, the shear and the vorticity allows to determine further characteristics of the plane wave space-time obtained in the Penrose limit, such as the occurence of caustics or singularities.

The expansion, the shear and the vorticity are defined in terms of the null vector $k^{\alpha}$ as follows, e.g., 12

$$
\begin{aligned}
\theta & =\frac{1}{2} k_{; \alpha}^{\alpha} \\
\sigma^{2} & =\frac{1}{2} k^{\alpha ; \beta} k_{(\alpha ; \beta)}-\frac{1}{4}\left(k_{; \alpha}^{\alpha}\right)^{2} \\
\omega_{\alpha \beta} \omega^{\alpha \beta} & =\frac{1}{2} k^{\alpha ; \beta} k_{[\alpha ; \beta]},
\end{aligned}
$$

where $\sigma^{2} \equiv \frac{1}{2} \sigma_{\alpha \beta} \sigma^{\alpha \beta}$.

In the Penrose adapted coordinates (cf. metric (2.1)) the tangent vector along the congruence of null geodesics is given by the vector $n^{\mu}$ of the null tetrad introduced in the previous section. The expansion, shear and vorticity in the original space-time with metric (2.1) are given by

$$
\begin{aligned}
\theta & =-\frac{1}{2} \mu_{1, u} \\
\sigma^{2} & =\frac{Z_{, u} \bar{Z}_{, u}}{(Z+\bar{Z})^{2}} \\
\omega_{\alpha \beta} \omega^{\alpha \beta} & =0 .
\end{aligned}
$$

There is no vorticity by construction of the coordinate system (cf equation (2.1) ) [1]. 
Introducing the new coordinates and rescaling the metric accordingly (cf equations (2.3)-(2.5)), shows that the quantities (3.26)-(3.28) are independent of the parameter $\Omega$. Hence expansion, shear and vorticity are the ones characterizing the congruence of null geodesics in the original space-time (2.1). This is consistent with the fact that only the transverse part of the metric enters into these expressions. The Raychaudhuri equation (3.25) determines the evolution of the expansion $\theta$. However, in the case of the plane wave space-time resulting from taking the Penrose limit, the kinematic quantities expansion, shear and vorticity are already known since they are directly derived from the original space-time. Thus it can be immediately determined if any of these quantities becomes unbounded at some point and thus a singularity develops.

As an example a Kasner type metric will be considered. The metric is given by

$$
d s^{2}=d t^{2}-t^{2 p_{1}} d x^{2}-t^{2 p_{2}} d y^{2}-t^{2 p_{3}} d z^{2}
$$

where the Kasner exponents $p_{i}$ satisfy the relations $\sum_{i=1}^{3} p_{i}=1$. In vacuum there is an additional relation, that is, $\sum_{i=1}^{3} p_{i}^{2}=1$. For stiff perfect fluid cosmologies the righthandside of this relation is equal to a constant unequal to 1 .

Kasner type metrics provide a good approximation for the description of space-times close to a singularity. Furthermore, for spatially homogeneous models it has been shown that there is a class of models for which the approach to the singularity is oscillatory and chaotic. The dynamics can be described by a succession of epochs in which the Kasner indices are interchanged [15].

Introducing conformal time $T$, defining null coordinates $u=T-z, v=T+z$ taking the Penrose limit according to (2.3)-(2.6) and redefining $u$ and rescaling the remaining coordinates accordingly, the resulting plane wave space-time is given by

$$
d s^{2}=d u d v-u^{\alpha_{1}} d x^{2}-u^{\alpha_{2}} d y^{2},
$$

where $\alpha_{1} \equiv \frac{2 p_{1}}{2-\left(p_{1}+p_{2}\right)}$ and $\alpha_{2} \equiv \frac{2 p_{2}}{2-\left(p_{1}+p_{2}\right)}$. Determining $\mu_{1}$ and $Z$ results in

$$
\mu_{1}=-\frac{\alpha_{1}+\alpha_{2}}{2} \ln u \quad Z=u^{\frac{\alpha_{2}-\alpha_{1}}{2}} .
$$

Thus the expansion and the shear are given by

$$
\begin{aligned}
\theta & =\frac{\alpha_{1}+\alpha_{2}}{4} \frac{1}{u} \\
\sigma^{2} & =\frac{1}{4}\left(\frac{\alpha_{2}-\alpha_{1}}{2}\right)^{2} \frac{1}{u^{2}} .
\end{aligned}
$$

In spatially homogeneous space-times the ratio of shear over the expansion is a measure of anisotropy. Calculating this ratio in the case of the null geodesics it is found that

$$
\frac{\sigma^{2}}{\theta^{2}}=\left(\frac{\alpha_{2}-\alpha_{1}}{\alpha_{1}+\alpha_{2}}\right)^{2}=\left(\frac{p_{2}-p_{1}}{p_{1}+p_{2}}\right)^{2} .
$$

Thus the expansion $\theta$ and the shear $\sigma^{2}$ both diverge as $u \rightarrow 0$. However, their ratio is constant. Thus the amount of anisotropy measured by the ratio $\sigma^{2} / \theta^{2}$ equals a constant, determined by the Kasner exponents in the $x$ - and $y$ - directions. The amount of anisotropy is not changed by taking the Penrose limit, that is, it is independent of the parameter $\Omega$. 
In comparison, the anisotropy along a congruence of timelike geodesics is determined by the constant ratio

$$
\frac{\sigma^{2}}{\theta^{2}}=\frac{1}{3}\left(\sum_{i} p_{i}^{2}-p_{1} p_{2}-p_{1} p_{3}-p_{2} p_{3}\right) .
$$

Spatially homogeneous space-times with form fields [16] as well as vacuum Bianchi IX spacetimes show chaotic behaviour in the approach to the initial singularity. This behaviour is well described by a succession of Kasner epochs, each determined by a different set of Kasner exponents $\left(p_{1}, p_{2}, p_{3}\right)$. Einstein's equations cause the effective change in the Kasner exponents. However, in the case of the Penrose limit the approach to the null singularity at $u=0$ is simpler than in the case of the approach to the space-like singularity of the original space-time at $t=0$. The initial set of Kasner exponents $\left(p_{1}, p_{2}, p_{3}\right)$ remains unchanged, since the only Einstein equation of a plane wave is given by

$$
2 \mu_{1, u u}-\mu_{1, u}^{2}-4 \frac{Z_{, u} \bar{Z}_{, u}}{(Z+\bar{Z})^{2}}=4 \Phi_{22},
$$

which in vacuum is satisfied by metrics of type (3.30) with $\sum_{i} p_{i}=1=\sum_{i} p_{i}^{2}$. The disappearance of the oscillating behaviour in the plane wave space-time obtained in the Penrose limit might also be understood from the point of view of functional genericity of the metric. General vacuum Bianchi IX space-times, for example, are described by 8 free functions. In the case of metrics of the type of equation (2.1) it has been argued that the most general solution of this type has 8 arbitrary functions [17. However, taking the Penrose limit of this metric results in the vanishing of the $V V$-, the $X V$ - and $Y V$ - components. Thus the resulting plane wave space-time has less degrees of freedom than the general type of the original space-time. Since chaotic behaviour in the approach to the singularity is related to the functional genericity of the space-time any chaotic behaviour of the original space-time is not expected to persist in the Penrose limit.

\section{Conclusions}

The components of the curvature tensor are known to show a peeling-off behaviour in the case of the space-time of an isolated radiating source or in the case of space-times admitting two abelian Killing vectors [3, 4, 5]. In these cases a plane wave space-time is approached as a global limit of the space-time. Therefore, the peeling-off behaviour manifests itself as an expansion in one of the coordinates of the space-time.

Here, it has been shown that in the Penrose limit the components of the Weyl tensor as well as those of the energy-momentum tensor have a peeling-off property. The Penrose limit results locally, in the neighbourhood of a particular null geodesic, in a plane wave space-time. In order to obtain the Penrose limit the coordinates and the metric are rescaled by a constant parameter $\Omega$. It is exactly this parameter which controlls the peeling-off behaviour of the curvature components as well as those of the energy-momentum tensor.

The approach taken here is in the line of the covariant approach to the Penrose limit [9, 10]. Using the Newman-Penrose formalism it has been shown that the component $\Psi_{4}$ of the Weyl tensor of the original metric in coordinates adapted to the neighbourhood around a null geodesic is exactly the one that remains the only non-vanishing component of the Weyl tensor in the resulting plane wave space-time. Similarly, $\Phi_{22}$ is the only non-vanishing component of the Ricci tensor. 
The peeling-off behaviour in the parameter $\Omega$ might be used to find corrections to the resulting plane wave space-time also with respect to the discussion of singularities in these type of spacetimes.

The expansion, shear and vorticity of the congruence of null geodesic around which the Penrose limit is taken has been determined. These expressions do not depend on the constant parameter $\Omega$. Their values are determined by functions characterizing the original metric. By construction of the coordinate system the congruence of null geodesics has vanishing vorticity.

A large class of cosmological solutions close to the initial singularity is well approximated by Kasner like space-times. For this type of metrics it has been found that the anisotropy as measured by the ratio of shear over the expansion is a constant. This is the case for congruences of time-like as well as null geodesics.

In the case of Bianchi IX vacuum and of spatially homogeneous models with form fields it is known that the approach to the initial (space-like) singularity is oscillatory and chaotic. The dynamics is described by the succession of epochs characterized by different Kasner exponents. Taking the Penrose limit in one of these epochs the corresponding Kasner indices remain unchanged. Therefore, the approach to the appearing null singularity in the plane wave space-time is not chaotic. This could also be interpreted in terms of the functional genericity of the plane wave space-time resulting in the Penrose limit.

\section{Acknowledgements}

This work has been supported by the programme "Ramón y Cajal" of the M.E.C. (Spain). Partial support by Spanish Science Ministry Grant FPA 2002-02037 is acknowledged.

\section{Appendix: Quantities in the Newman-Penrose formalism}

Several quantities in the Newman-Penrose formalism are given (cf, e.g., [12]). The tetrad metric is given by

$$
\eta_{(a)(b)}=\eta^{(a)(b)}=\left(\begin{array}{cccc}
0 & 1 & 0 & 0 \\
1 & 0 & 0 & 0 \\
0 & 0 & 0 & -1 \\
0 & 0 & -1 & 0
\end{array}\right)
$$

Tetrad indices are enclosed in brackets ( ). They run from 1 to 4.

For a space-time with line element (2.1) a null tetrad is provided by

$$
\begin{aligned}
e_{(1)}=e^{(2)} & =l_{\mu}=\left(\begin{array}{llll}
1 & \frac{\alpha}{2} & \beta_{1} & \beta_{2}
\end{array}\right) \\
e_{(2)}=e^{(1)} & =n_{\mu}=\left(\begin{array}{llll}
0 & 1 & 0 & 0
\end{array}\right) \\
e_{(3)}=-e^{(4)} & =m_{\mu}=\left(\begin{array}{llll}
0 & 0 & -\frac{e^{-\frac{\mu_{1}}{2}}}{(Z+\bar{Z})^{\frac{1}{2}}} & \frac{i \bar{Z} e^{-\frac{\mu_{1}}{2}}}{(Z+\bar{Z})^{\frac{1}{2}}}
\end{array}\right) \\
e_{(4)}=-e^{(3)} & =\bar{m}_{\mu}=\left(\begin{array}{llll}
0 & 0 & -\frac{e^{-\frac{\mu_{1}}{2}}}{(Z+\bar{Z})^{\frac{1}{2}}} & -\frac{i Z e^{-\frac{\mu_{1}}{2}}}{(Z+\bar{Z})^{\frac{1}{2}}}
\end{array}\right) .
\end{aligned}
$$


The corresponding contravariant components are given by

$$
\begin{aligned}
l^{\mu} & =\left(\begin{array}{llll}
-\frac{\alpha}{2} & 1 & 0 & 0
\end{array}\right) \\
n^{\mu} & =\left(\begin{array}{llll}
1 & 0 & 0 & 0
\end{array}\right) \\
m^{\mu} & =\left(\begin{array}{llll}
-\frac{e^{\frac{\mu_{1}}{2}}}{(Z+\bar{Z})^{\frac{1}{2}}}\left(\beta_{1} \bar{Z}-i \beta_{2}\right) & 0 & \frac{e^{\frac{\mu_{1}}{2}}}{(Z+\bar{Z})^{\frac{1}{2}}} \bar{Z} & -i \frac{e^{\frac{\mu_{1}}{2}}}{(Z+\bar{Z})^{\frac{1}{2}}}
\end{array}\right) \\
\bar{m}^{\mu} & =\left(\begin{array}{llll}
-\frac{e^{\frac{\mu_{1}}{2}}}{(Z+\bar{Z})^{\frac{1}{2}}}\left(\beta_{1} Z+i \beta_{2}\right) & 0 & \frac{e^{\frac{\mu_{1}}{2}}}{(Z+\bar{Z})^{\frac{1}{2}}} Z & i \frac{e^{\frac{\mu_{1}}{2}}}{(Z+\bar{Z})^{\frac{1}{2}}}
\end{array}\right) .
\end{aligned}
$$

The Newman-Penrose spin coefficients derived from the Ricci rotation coefficients, $\gamma_{(a)(b)(c)}=e_{(a)}^{\mu} e_{(b) \mu ; \nu} e_{(c)}^{\nu}$, are given by

$$
\begin{aligned}
& \kappa=\frac{e^{\frac{\mu_{1}}{2}}}{(Z+\bar{Z})^{\frac{1}{2}}}\left[\bar{Z}\left(\frac{1}{2} \beta_{1} \alpha_{, u}-\frac{1}{2} \alpha \beta_{1, u}+\beta_{1, v}-\frac{1}{2} \alpha_{, x}\right)-i\left(\frac{1}{2} \alpha_{, u} \beta_{2}-\frac{1}{2} \alpha \beta_{2, u}+\beta_{2, v}-\frac{1}{2} \alpha_{, y}\right)\right] \\
& \sigma=\frac{-\frac{\alpha}{2} \bar{Z}_{, u}+\bar{Z}_{, v}}{Z+\bar{Z}} \\
& \lambda=-\frac{Z_{, u}}{Z+\bar{Z}} \\
& \nu=0 \\
& \rho=\frac{1}{2}\left[\mu_{1, v}-\frac{\alpha}{2} \mu_{1, u}+i e^{\mu_{1}}\left(\beta_{1} \beta_{2, u}-\beta_{2} \beta_{1, u}+\beta_{1, y}-\beta_{2, x}\right)\right] \\
& \mu=-\frac{1}{2} \mu_{1, u} \\
& \tau=\frac{1}{2} \frac{e^{\frac{\mu_{1}}{2}}}{\sqrt{Z+\bar{Z}}}\left(\beta_{1, u} \bar{Z}-i \beta_{2, u}\right) \\
& \pi=-\frac{1}{2} \frac{e^{\frac{\mu_{1}}{2}}}{\sqrt{Z+Z}}\left(\beta_{1, u} Z+i \beta_{2, u}\right) \\
& \epsilon=\frac{1}{4}\left[-\frac{Z_{, v}-\bar{Z}_{, v}}{Z+\bar{Z}}+\frac{\alpha}{2} \frac{Z_{, u}-\bar{Z}_{, u}}{Z+\bar{Z}}+i e^{\mu_{1}}\left[\beta_{1} \beta_{2, u}-\beta_{2} \beta_{1, u}+\beta_{1, y}-\beta_{2, x}\right]-\alpha_{, u}\right] \\
& \gamma=\frac{1}{4} \frac{\bar{Z}_{, u}-Z_{, u}}{Z+\bar{Z}} \\
& \alpha_{N P}=-\frac{1}{2}\left[\frac{1}{2} \frac{e^{\frac{\mu_{1}}{2}}}{\sqrt{Z+Z}}\left(\beta_{1, u} Z+i \beta_{2, u}\right)+i e^{\mu_{1}}\left[-\left(\frac{e^{-\frac{\mu_{1}}{2}}}{\sqrt{Z+Z}}\right)_{, u} \beta_{2}+i\left(\frac{Z e^{-\frac{\mu_{1}}{2}}}{\sqrt{Z+Z}}\right)_{, u} \beta_{1}\right.\right. \\
& \left.\left.+\left(\frac{e^{-\frac{\mu_{1}}{2}}}{\sqrt{Z+\bar{Z}}}\right)_{, y}-i\left(\frac{Z e^{-\frac{\mu_{1}}{2}}}{\sqrt{Z+\bar{Z}}}\right)_{, x}\right]\right] \\
& \beta=-\frac{1}{2}\left[\frac{1}{2} \frac{e^{\frac{\mu_{1}}{2}}}{\sqrt{Z+\bar{Z}}}\left(\beta_{1, u} \bar{Z}-i \beta_{2, u}\right)+i e^{\mu_{1}}\left[-\left(\frac{e^{-\frac{\mu_{1}}{2}}}{\sqrt{Z+\bar{Z}}}\right)_{, u} \beta_{2}-i\left(\frac{\bar{Z} e^{-\frac{\mu_{1}}{2}}}{\sqrt{Z+\bar{Z}}}\right)_{, u} \beta_{1}\right.\right. \\
& \left.\left.+\left(\frac{e^{-\frac{\mu_{1}}{2}}}{\sqrt{Z+\bar{Z}}}\right)_{, y}+i\left(\frac{\bar{Z} e^{-\frac{\mu_{1}}{2}}}{\sqrt{Z+\bar{Z}}}\right)_{, x}\right]\right] \text {, }
\end{aligned}
$$


where, $u$ denotes $\frac{\partial}{\partial u}$ etc.

The directional derivatives for the metric (2.1) are given by

$$
\begin{aligned}
D & =e_{(1)}=e^{(2)}=-\frac{\alpha}{2} \frac{\partial}{\partial u}+\frac{\partial}{\partial v} \\
\Delta & =e_{(2)}=e^{(1)}=\frac{\partial}{\partial u} \\
\delta & =e_{(3)}=-e^{(4)}=-\frac{e^{\frac{\mu_{1}}{2}}}{\sqrt{Z+\bar{Z}}}\left(\beta_{1} \bar{Z}-i \beta_{2}\right) \frac{\partial}{\partial u}+\frac{e^{\frac{\mu_{1}}{2}}}{\sqrt{Z+\bar{Z}}} \bar{Z} \frac{\partial}{\partial x}-i \frac{e^{\frac{\mu_{1}}{2}}}{\sqrt{Z+\bar{Z}}} \frac{\partial}{\partial y} \\
\bar{\delta} & =e_{(4)}=-e^{(3)}=-\frac{e^{\frac{\mu_{1}}{2}}}{\sqrt{Z+\bar{Z}}}\left(\beta_{1} Z+i \beta_{2}\right) \frac{\partial}{\partial u}+\frac{e^{\frac{\mu_{1}}{2}}}{\sqrt{Z+\bar{Z}}} Z \frac{\partial}{\partial x}+i \frac{e^{\frac{\mu_{1}}{2}}}{\sqrt{Z+\bar{Z}}} \frac{\partial}{\partial y} .
\end{aligned}
$$

The components of the Weyl tensor are given by

$$
\begin{aligned}
& \Psi_{0}=-C_{(1)(3)(1)(3)}=-C_{\mu \nu \lambda \kappa} l^{\mu} m^{\nu} l^{\lambda} m^{\kappa} \\
& \Psi_{1}=-C_{(1)(2)(1)(3)}=-C_{\mu \nu \lambda \kappa} l^{\mu} n^{\nu} l^{\lambda} m^{\kappa} \\
& \Psi_{2}=-C_{(1)(3)(4)(2)}=-C_{\mu \nu \lambda \kappa} l^{\mu} m^{\nu} \bar{m}^{\lambda} n^{\kappa} \\
& \Psi_{3}=-C_{(1)(2)(4)(2)}=-C_{\mu \nu \lambda \kappa} l^{\mu} n^{\nu} \bar{m}^{\lambda} n^{\kappa} \\
& \Psi_{4}=-C_{(2)(4)(2)(4)}=-C_{\mu \nu \lambda \kappa} n^{\mu} \bar{m}^{\nu} n^{\lambda} \bar{m}^{\kappa}
\end{aligned}
$$

The components of the Ricci tensor are denoted as

$$
\begin{array}{ll}
\Phi_{00}=-\frac{1}{2} R_{(1)(1)} & \Phi_{22}=-\frac{1}{2} R_{(2)(2)} \\
\Phi_{02}=-\frac{1}{2} R_{(3)(3)} & \Phi_{20}=-\frac{1}{2} R_{(4)(4)} \\
\Phi_{11}=-\frac{1}{4}\left(R_{(1)(2)}+R_{(3)(4)}\right) & \Phi_{01}=-\frac{1}{2} R_{(1)(3)} \\
\Lambda=\frac{1}{24} R=\frac{1}{12}\left(R_{(1)(2)}-R_{(3)(4)}\right) & \Phi_{10}=-\frac{1}{2} R_{(1)(4)} \\
\Phi_{12}=-\frac{1}{2} R_{(2)(3)} & \Phi_{21}=-\frac{1}{2} R_{(2)(4)}
\end{array}
$$

\section{References}

[1] R. Penrose, "Any space-time has a plane wave as a limit," in Differential geometry and relativity, ed. M. Flato, Reidel, Dordrecht (1976).

[2] M. Blau, J. Figueroa-O'Farrill and G. Papadopoulos, Class. Quant. Grav. 19 (2002) 4753.

[3] H. Bondi, M. G. H. van der Burg, A. W. K. Metzner, Proc. R. Soc. A 269 (1962) 21; R. K. Sachs, Proc. R. Soc. A 270 (1962) 103; E. T. Newman and R. Penrose, J. Math. Phys. 3 (1962) 566; R. K. Sachs (1964) in Relativity, Groups and Topology ed. C. DeWitt and B. DeWitt (New York: Gordon and Breach).

[4] J. Stachel, J. Math. Phys. 7 (1966) 1321; M. Carmeli and A. Feinstein, Phys. Lett. A 103 (1984) 318; Int. J. Theor. Phys. 24 (1985) 1009; K. E. Kunze, Class. Quantum Grav. 16 (1999) 3795 .

[5] G. F. Bressange and P. A. Hogan, Phys. Rev. D 60 (1999) 104006.

[6] D. Amati and C. Klimčik, Phys. Lett. B 219 (1989) 443. 
[7] J. B. Griffiths, "Colliding Plane Waves In General Relativity," Oxford, UK: Clarendon (1991).

[8] K. E. Kunze, Phys. Rev. D 68 (2003) 063517.

[9] M. Blau, M. Borunda, M. O'Loughlin and G. Papadopoulos, Class. Quant. Grav. 21 (2004) L43.

[10] M. Blau, M. Borunda, M. O’Loughlin and G. Papadopoulos, JHEP 0407 (2004) 068.

[11] G. Papadopoulos, "Power-law singularities in string theory and M-theory," arXiv:hep-th/0404172.

[12] S. Chandrasekhar, " The Mathematical Theory of Black Holes," Oxford: Oxford University Press, (1992).

[13] J. Stewart, "Advanced general relativity", Cambridge, UK: Cambridge University Press (1991).

[14] E. Poisson, "A Relativist's Toolkit", Cambridge, UK: Cambridge University Press (2004).

[15] C.W. Misner, Phys. Rev. Lett. 22 (1969) 1071; V.A. Belinsky, I.M. Khalatnikov and E.M. Lifshitz, Adv. Phys. 19 (1970) 525; J.D. Barrow, Phys. Rep. 85 (1982) 1.

[16] V. G. LeBlanc, D. Kerr and J. Wainwright, Class. Quant. Grav. 12 (1995) 513; T. Damour and M. Henneaux, Gen. Rel. Grav. 32 (2000) 2339.

[17] A. Ori and E. E. Flanagan, Phys. Rev. D 53 (1996) 1754. 